\title{
Subdiffusive fluctuations of "pulled" fronts with multiplicative noise
}

\author{
Andrea Rocco, ${ }^{1}$ Ute Ebert, ${ }^{2}$ and Wim van Saarloos ${ }^{3}$ \\ ${ }^{1}$ Departament ECM, Facultat de Física, Universitat de Barcelona, Avenida Diagonal 647, E-08028 Barcelona, Spain \\ ${ }^{2}$ Centrum voor Wiskunde en Informatica, Postbus 94079, 1090 GB Amsterdam, The Netherlands \\ ${ }^{3}$ Instituut-Lorentz, Universiteit Leiden, Postbus 9506, 2300 RA Leiden, The Netherlands
}

(Received 6 April 2000)

\begin{abstract}
We study the propagation of a "pulled" front with multiplicative noise that is created by a local perturbation of an unstable state. Unlike a front propagating into a metastable state, where a separation of time scales for sufficiently large $t$ creates a diffusive wandering of the front position about its mean, we predict that for so-called pulled fronts, the fluctuations are subdiffusive with root mean square wandering $\Delta(t) \sim t^{1 / 4}$, not $t^{1 / 2}$. The subdiffusive behavior is confirmed by numerical simulations: For $t \leqslant 600$, these yield an effective exponent slightly larger than $1 / 4$.

PACS number(s): 05.40. $-\mathrm{a}, 47.54 .+\mathrm{r}$
\end{abstract}

Since the late 1930s, when the concept of front propagation emerged in the field of population dynamics [1,2], interest in this type of problems has been growing steadily in chemistry [3], physics [4], and mathematics [5]. In physics, the importance of the problem has become more and more clear since it plays a role in a large variety of situations, ranging from reaction-diffusion systems to pattern forming systems in general [6].

Front propagation into unstable states is an interesting dynamical problem by itself. For a front evolving from a local perturbation there are but two possible propagation mechanisms that are determined by the nonlinearities in the equation of motion: Either the nonlinearities determine the velocity of the front that then is called "pushed"; or the nonlinearities simply cause saturation and the velocity is determined by a linearization about the unstable state. Fronts of this type are called "pulled" because they are "pulled along" by the spreading and growth of small perturbations about the unstable state [7]. Hence, pulled front propagation can occur only if the penetrated state is linearly unstable. The pushed and pulled regimes are also known as nonlinear and linear marginal stability [8]. For the discussion below, it is important to realize that pushed fronts relax exponentially in time to their long time asymptotes, but that pulled fronts relax algebraically without characteristic time scale [7]. Hence, an adiabatic decoupling of some outer dynamics from the internal relaxation of a pulled front is not possible [9], and stochastic pulled fronts may show anomalous scaling [10].

Generally, noise can affect the phenomenological description of a reaction-diffusion system in various ways. A first possibility is intrinsic noise modelled typically by additive thermal noise in a Langevin type equation. A second possibility, on which the present paper is focused, is at the external level, e.g., due to fluctuations of some control parameter. An example are the fluctuations of the luminosity intensity in the photosensitive Belousov-Zhabotinsky reaction [11]. Such fluctuations enter the dynamical equation as multiplicative noise.

The multiplicative noise of the control parameter usually results in a modification of the mean propagation velocity of the front and in a stochastic wandering of the front position around its mean propagation. This means that the noisy front can be thought of as a coherent structure whose motion can be decomposed into drift plus Brownian motion, very much like a particle sedimenting in a fluid. The drift component corresponds to an average front, with the average taken over the ensemble of all the realizations of the noise. It propagates according to a deterministic equation of motion, whose dynamical parameters are in the simplest case just renormalized by the noise. Theoretically, the important question then arises whether the effects of the fluctuations of the front can be understood in terms of a diffusive or subdiffusive wandering of some suitably defined front position.

The renormalization of the front velocity has been studied in the pushed and pulled regime [12], while the wandering process is understood only in the pushed case [13], where it has been shown to be diffusive: the root mean square position of the front $\Delta$ grows with time as $\sqrt{2 D_{f} t}$. Actually, the expression for the effective front diffusion coefficient $D_{f}$ derived by Armero et al. [13] was found to break down for pulled fronts, and it was suggested that the wandering of pulled fronts is subdiffusive.

In this Rapid Communication we take up the issue of the stochastic wandering of pulled fronts about their mean position, and predict that in the presence of multiplicative noise pulled fronts behave subdiffusively, with $\Delta \sim t^{1 / 4}$. This prediction is based on two different arguments. First of all, we heuristically insert the leading edge asymptotics of the relaxing pulled front into the expression for the diffusion coefficient $D_{f}$ of pushed fronts, and immediately find $\Delta \sim t^{1 / 4}$. Our second argument for the subdiffusive $\Delta \sim t^{1 / 4}$ behavior comes from mapping the dynamically important region onto the KPZ equation. We finally also present data of extensive numerical simulations that support our analytical prediction that the wandering is subdiffusive with exponent close to $1 / 4$.

The qualitative difference between pushed and pulled fronts results from the fact that the dynamically important region for pushed fronts is the interior front region, whose extent is finite, while that of pulled fronts is the leading edge ahead of the front [7]. Starting from a local initial perturbation, the leading edge region grows without bound, and as we shall see, this causes the subdiffusive behavior. The 
power law relaxation of deterministic pulled fronts is another manifestation of the leading edge dominated dynamics of pulled fronts [7].

For concreteness, we derive our results by including noise in the one-dimensional prototype front equation

$$
\frac{\partial \phi}{\partial t}=D \frac{\partial^{2} \phi}{\partial x^{2}}+f(\phi), \quad f(\phi)=\phi(1-\phi)(a+\phi) .
$$

Here $a$ is a parameter which plays the role of the control parameter. Equation (1) has a stable state $\phi=1$ and a stationary state $\phi=0$ whose relative stability can be tuned by changing the value of the parameter $a$. The case $-\frac{1}{2}<a$ $<\frac{1}{2}$ leads to pushed dynamics, while $\frac{1}{2}<a<1$ produces pulled fronts [7]. For the case $a=1$, which we will study, the so-called Fisher-Kolmogoroff-Petrovsky-Piscounoff (FKPP) equation $[1,2]$ is recovered.

Let us assume now that the parameter $a$ is replaced by a new fluctuating parameter $a(x, t)$ with average $\bar{a}, a$ $\rightarrow a(x, t)=\bar{a}+\mu(x, t)$, where $\mu(x, t)$ is a Gaussian noise with the moments

$$
\begin{gathered}
\langle\mu(x, t)\rangle_{\mu}=0, \\
\left\langle\mu(x, t) \mu\left(x^{\prime}, t^{\prime}\right)\right\rangle_{\mu}=2 \varepsilon C\left(\lambda_{\mu}\left|x-x^{\prime}\right|\right) \delta\left(t-t^{\prime}\right),
\end{gathered}
$$

with $\int d x C\left(\lambda_{\mu},|x|\right)=1$. We interpret the stochastic partial differential equations (PDE) defined by Eqs. (1)-(3) in the Stratonovich sense [14]. Notice that if $1 / \lambda_{\mu}$ is much smaller than any other length scale in the system, the noise defined by the correlator (3) is effectively white in both time and space.

Since according to Eq. (1) $\phi$ converges to 1 and is noiseless behind the front, we can suitably define the position $\mathrm{x}_{f}(t)$ of a noisy front propagating to the right into the unstable state $\phi=0$ by

$$
\mathrm{x}_{f}(t)=\int_{0}^{\infty} d x \phi(x, t)
$$

The displacement $\Delta \mathrm{x}_{f}(t)=\mathrm{x}_{f}(t)-\mathrm{x}_{f}(0)$ on average grows with the noise renormalized mean velocity $\bar{v}_{R}=\left\langle\dot{\mathrm{x}}_{f}\right\rangle_{\mu}$. The fluctuations about the mean displacement $\left\langle\Delta \mathrm{x}_{f}(t)\right\rangle_{\mu}=\bar{v}_{R} t$ are measured by

$$
\Delta(t)=\sqrt{\left\langle\left[\Delta \mathrm{x}_{f}(t)-\left\langle\Delta \mathrm{x}_{f}(t)\right\rangle_{\mu}\right]^{2}\right\rangle_{\mu}} .
$$

If we relate $\Delta(t)$ to a diffusion coefficient $D_{f}$ by writing

$$
\Delta^{2}(t)=\int_{0}^{t} d t^{\prime} 2 D_{f}\left(t^{\prime}\right)
$$

then for pushed fronts the following expression for the diffusion coefficient $D_{f}$ can be derived $[13,15]$ :

$$
D_{f}=\varepsilon \frac{\int_{-\infty}^{\infty} d \xi e^{2 \bar{v}_{R} \xi}(d \bar{\phi} / d \xi)^{2} g^{2}(\bar{\phi})}{\left[\int_{-\infty}^{\infty} d \xi e^{\bar{v}_{R} \xi}(d \bar{\phi} / d \xi)^{2}\right]^{2}} .
$$

In this formula, $\bar{\phi}$ is the deterministic field associated with the front moving with the renormalized pushed velocity $\bar{v}_{R}, g(\bar{\phi})=\partial f /\left.\partial a\right|_{\bar{a}}$ is the derivative of the reaction term with respect to the control parameter, and $\xi=x-\bar{v}_{R} t$ is the comoving coordinate.

For pushed fronts, $D_{f}$ given by Eq. (7) is finite and timeindependent, and hence this gives the diffusive behavior $\Delta^{2}(t)=2 D_{f} t$. This means that on sufficiently long time scales the random displacement is approximately Markovian, i.e., the sum of uncorrelated and equally distributed random displacements on shorter time scales.

As an example of a pulled front with multiplicative noise, we now study the case $\bar{a}=1$ :

$$
\frac{\partial \phi}{\partial t}=D \frac{\partial^{2} \phi}{\partial x^{2}}+\phi+\mu \phi-\mu \phi^{2}-\phi^{3} .
$$

The noise renormalized mean velocity $\bar{v}_{R}^{*}$ of the pulled front can be calculated explicitly [12]:

$$
\bar{v}_{R}^{*}=\langle\dot{\mathrm{x}}(t)\rangle_{\mu}=2 \sqrt{D[1+\varepsilon C(0)]} .
$$

However, it is immediately clear that the fluctuation formula (7) cannot naively be extended to the pulled regime.

First of all, for a pulled front the expression (7) simply diverges. The divergence of solvability-type expressions actually holds more generally for perturbative expansions about a pulled front [9]. For a pulled front, the dynamically important region is the leading edge defined as the region where linearization about the unstable state is a valid approximation; the fact that solvability-type integrals like Eq. (7) diverge there reflects that the dynamically important region becomes semi-infinite.

Second, a pulled front has no characteristic relaxation time [7], so there is no reason for the Markovian approximation underlying diffusive wandering. Rather the leading edge relaxes asymptotically as [7]

$$
\begin{gathered}
\phi \approx \alpha \xi_{R} e^{-\lambda_{R}^{*} \xi_{R}} e^{-\xi_{R}^{2} / 4 D t} / t^{3 / 2}, \quad \lambda_{R}^{*}=\bar{v}_{R}^{*} / 2, \\
\text { for } \xi_{R}=x-\bar{v}_{R}^{*} t \gg 1 \text { and } t \gg 1 .
\end{gathered}
$$

The presence of the $\alpha \xi_{R} / t^{3 / 2}$ term in front of the exponentials is actually the fingerprint of the full equation being nonlinear. The expression (10) defines a time-dependent Gaussian cutoff $\xi_{c} \sim \sqrt{4 D t}$, which regularizes the integrals in Eq. (7). In fact, the evaluation of Eq. (7) with Eq. (10) yields

$$
D_{f}(t) \approx \frac{3 \varepsilon}{\left(\bar{v}_{R}^{*}\right)^{2} \sqrt{\pi D}} \frac{1}{\sqrt{t}} \quad(t \gg 1) .
$$

Notice that for large times $D_{f}(t)$ vanishes, marking the nondiffusive wandering of pulled fronts. Insertion into Eq. (6) yields

$$
\Delta(t)=\sqrt{2 \int_{0}^{t} d t^{\prime} D_{f}\left(t^{\prime}\right)} \approx\left(\frac{12 \varepsilon}{\left(\bar{v}_{R}^{*}\right)^{2} \sqrt{\pi D}}\right)^{1 / 2} t^{1 / 4}
$$


so the fluctuations are subdiffusive with exponent $1 / 4$ rather than $1 / 2$.

Although the above argument does capture the essential features of fluctuating pulled fronts, it is not entirely systematic, as it is based on the extrapolation of the solvability condition (7) to the pulled regime.

In order to substantiate the scaling $\Delta(t) \sim t^{1 / 4}$ for a relaxing pulled front with a time-dependent analysis, let us go back to Eq. (8). The leading edge region can be studied by means of the leading edge transformation,

$$
\begin{gathered}
\phi(x, t)=\psi(\xi, t) e^{-\lambda * \xi}, \\
\xi=x-v^{*} t, \quad v^{*}=2, \quad \lambda^{*}=1 .
\end{gathered}
$$

Equation (8) can then be written as

$$
\frac{\partial \psi}{\partial t}=D \frac{\partial^{2} \psi}{\partial \xi^{2}}-\psi+e^{\xi}\left[(1+\mu) \psi e^{-\xi}-\mu \psi^{2} e^{-2 \xi}-\psi^{3} e^{-3 \xi}\right]
$$

For $\xi \gg 1$, the nonlinearities can be neglected,

$$
\frac{\partial \psi}{\partial t}=D \frac{\partial^{2} \psi}{\partial \xi^{2}}+\mu \psi, \text { for } \xi \gg 1
$$

Notice that the noise in this "directed polymer" equation still is multiplicative. The Cole-Hopf transformation

$$
\psi(\xi, t)=e^{h(\xi, t)},
$$

converts Eq. (15) into an equation with additive noise:

$$
\frac{\partial h}{\partial t}=D \frac{\partial^{2} h}{\partial \xi^{2}}+D\left(\frac{\partial h}{\partial \xi}\right)^{2}+\mu, \text { for } \xi \gg 1
$$

Equation (17) is the celebrated one-dimensional Kardar Parisi Zhang (KPZ) interface equation [16].

The essential difference between our problem and previous studies of the KPZ equation are the initial and boundary conditions. After some temporal evolution, the nonlinearities in the original $\phi$ equation will lead to the fluctuationless saturation of $\phi$ at the value of unity for $\xi \ll-1$, which corresponds to the fluctuationless slope $h \approx \lambda * \xi$ behind the front: It is as if the KPZ equation has to be solved in the positive half-space with (roughly) a fixed boundary. On the other hand, by translating Eq. (10) back into $h$, we see that for large $\xi$ and $t$, the average interface shape $h_{a v}$ should be given by

$$
h_{a v} \approx \ln \left(\alpha \xi_{R} / t^{3 / 2}\right)+\lambda * \xi-\lambda{ }_{R}^{*} \xi_{R}-\xi_{R}^{2} / 4 D t .
$$

Thus, apart from the logarithmic term the average interface is essentially tilted but flat up to the time-dependent crossover $\xi_{c} \approx \sqrt{4 D t}$ [17], and beyond $\xi_{c}$ it has the shape of a downward curved parabola with time dependent curvature. Together with the fact that the nonlinear term in Eq. (17) gives an average nonzero growth velocity, this makes the problem into a nonstandard fluctuating interface problem. Our central approximation is now to consider the relaxing front in the essentially straight but fluctuating section between 0 and $\sqrt{4 D t}$ as a KPZ interface with time-dependent length $L$

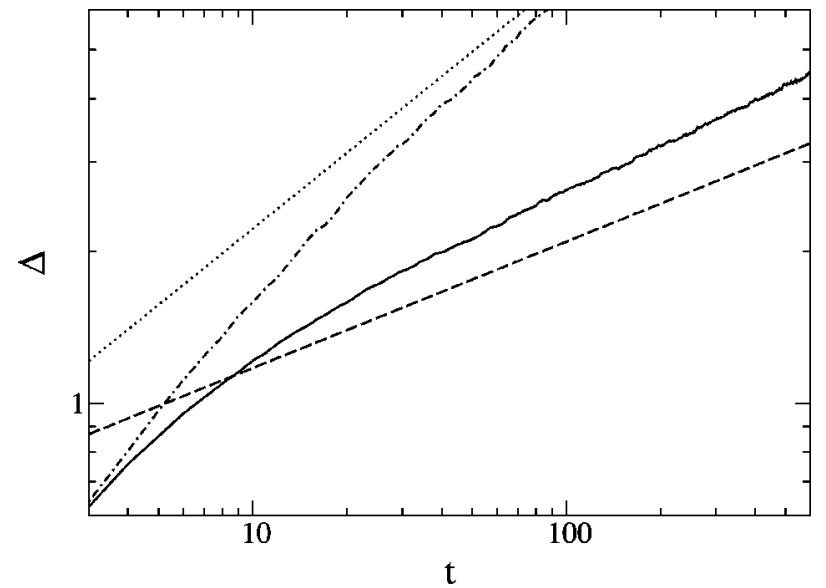

FIG. 1. Diffusive and subdiffusive spreading of the front position. The dotted-dashed curve correponds to the pushed case ( $a$ $=-0.3)$ and the solid one corresponds to the pulled case $(a=1)$. The dashed straight line is the prediction (12), while the dotted line indicates a slope $1 / 2$.

$=\mathcal{O}\left(\xi_{c}\right)$. As the scaling exponents of the KPZ equation are robust with respect to a geometric change of the fluctuating surface [18], we use the KPZ scaling functions for the root mean square width $W$ of the interface $h$,

$$
W(L, t)=t^{\beta} Y\left(\frac{t}{L^{z}}\right), \quad \beta=1 / 3, \quad z=3 / 2,
$$

where $W=\sqrt{\left\langle\overline{h(x, t)-\bar{h}(x, t)^{2}}\right\rangle_{\mu}}$, with the bar denoting a spatial average. The scaling function $Y(s)$ will depend on the shape of the roughening surface, but always has the limits $Y(s) \rightarrow s^{-\beta}$ for $s \rightarrow \infty, \quad Y(0) \approx$ const.

Inserting our approximation $L \sim \sqrt{t}$, we get

$$
W(L, t) \sim L^{z \beta} \sim(\sqrt{t})^{z \beta}=t^{1 / 4} .
$$

The final step of our argument is to convert this result into a prediction for the fluctuations of the front position. If we measure the position of the front by tracking a certain height $c, \phi\left(x_{c}, t\right)=$ const $=c$, and use the relations (13) and (16), we find

$$
\phi\left(x_{c}, t\right)=e^{-\lambda_{R}^{*}\left(x_{c}-\bar{v}_{R}^{*} t\right)+h}=\mathrm{const}=c .
$$

This implies that fluctuations in $h$ are just identical with fluctuations in $x_{c}$. Therefore, we get

$$
\Delta(t) \sim t^{1 / 4}
$$

which reproduces the scaling of our previous result (12).

We have also performed numerical simulations of the noisy front equation (1) with $a=-0.3$ (pushed) and $a=1$ [pulled, FKPP equation (8)] following the lines of [13]. The initial condition was taken as a step function $\phi(x, 0)=\theta\left(x_{0}\right.$ $-x)$. The numerical integration has been performed using a standard explicit Euler algorithm, in both cases the value of the noise was set to $\varepsilon=0.5$, and the zero value of the spatial noise correlator $C(0)$ was chosen as the inverse spatial integration mesh, $C(0)=1 / \Delta x$ [13]. The result is shown in Fig. 1 , where the function $\Delta(t)$ is plotted in both the pushed and 
the pulled case.

The specific features of the pulled regime make the problem quite delicate from the numerical point of view. In order to minimize finite size effects, which are particularly worrisome in this regime [7], we have worked with a large system size $(L=3000)$ and gridsize $\Delta x=1$ (the change in $v^{*}$ and $D$ due to the finite gridsize effect was taken into account following the prescription of [7]). This made sure that even at time $t=600$, the leading edge of the front never reached the boundary of the system.

We have also checked our program and system size extensively both for deterministic and noisy fronts, taking into account grid and time step effects according to [7].

Our final result, based on averaging over 10000 front realizations, is shown in Fig. 1; it clearly confirms the subdiffusive behavior predicted by our analytical arguments. Quantitatively, when we associate a single effective exponent with the late time slope in the log-log plot of Fig. 1, we get an effective exponent of about 0.29 rather than $1 / 4$. Over the time interval we have studied, the actual value of $\Delta(t)$ is somewhat larger than an asymptotic prediction (12), which is indicated with a dashed line. This may be due to the fact that Eq. (12) only gives the behavior for such long times that the time integral is dominated by its large $t$ behavior. The fact that $\Delta$ is only of the order of 4 at our latest times suggests that this asymptotic regime is only reached at very late times. Indeed, assuming that finite size effects are negligible, we attribute the fact that the effective exponent is slightly larger than 1/4 to the presence of slow crossovers, which surely are present in the system. Some of these can be estimated, while others are more difficult to trace. (i) We already noticed previously that we are actually dealing with a slightly curved KPZ interface, for which the crossover scaling functions are not known, and that the way in which the cutoff $\xi_{c}$ $=\mathcal{O}(\sqrt{t})$ enters the KPZ analysis requires further study. (ii) The corrections to our asymptotic estimates for the integrals in Eq. (7) are all of order $1 / \sqrt{t}$, with possible logarithmic corrections [7]. This indicates that the corrections to the scaling $\Delta \sim t^{1 / 4}$ are of order $t^{-1 / 4}$, possibly with logarithmic corrections. (iii) If initially $\phi$ falls off as $\exp \left(-\lambda_{R}^{*} x\right)$, then the associated KPZ interface remains straight towards $\xi=\infty$. For this case the KPZ scaling predicts $\Delta \sim t^{1 / 3}$. Presumably a crossover between exponent $1 / 3$ and $1 / 4$ could be present when starting with an initial condition slightly faster decaying than $\exp \left(-\lambda_{R}^{*} x\right)$. The identification of such a crossover and the modification of the global exponent due to these special initial conditions is an issue that will be addressed elsewhere.

We finally stress that our results apply to a much larger class of equations than nonlinear diffusion equations (1). The methods of generalization are analogous to those of $[7,9]$; a closely related result is the general argument put forward in [10] that noisy pulled fronts in more than one dimension should not obey KPZ scaling.

We thank J. Casademunt and L. Schäfer for useful discussions. A.R. thanks the Instituut-Lorentz for kind hospitality. He was supported by the European Commission Project No. ERBFMRX-CT96-0085 and U.E. by the Dutch Science Foundation NWO.
[1] R.A. Fisher, Ann. Eugenics 7, 355 (1937).

[2] A. Kolmogorov, I. Petrovsky, and N. Piskunov, Bull. Univ. Moskou Ser. Int. Se. A 1(6), 1 (1937).

[3] P. Fife, in Mathematical Aspects of Reacting and Diffusing Systems, edited by S. Levin, Lecture Notes in Biomathematics Vol. 28 (Springer-Verlag, New York, 1979).

[4] G. Dee and J.S. Langer, Phys. Rev. Lett. 50, 383 (1983).

[5] D.G. Aronson and H.F. Weinberger, Adv. Math. 30, 33 (1978).

[6] For a review, see, e.g., M.C. Cross and P.C. Hohenberg, Rev. Mod. Phys. 65, 851 (1993).

[7] U. Ebert and W. van Saarloos, Phys. Rev. Lett. 80, 1650 (1998); Physica D (to be published) (e-print cond-mat/ 0003181).

[8] W. van Saarloos, Phys. Rev. A 37, 211 (1988); 39, 6367 (1989).

[9] U. Ebert and W. van Saarloos, Phys. Rep. (to be published) (e-print cond-mat/0003184).

[10] G. Tripathy and W. van Saarloos, e-print cond-mat/0005513.

[11] I. Sendiña-Nadal, A.P. Munuzuri, D. Vives, V. Pérez-
Muñuzuri, J. Casademunt, L. Ramírez-Piscina, J.M. Sancho, and F. Sagues, Phys. Rev. Lett. 80, 5437 (1998).

[12] J. Armero, J.M. Sancho, J. Casademunt, A.M. Lacasta, L. Ramírez-Piscina, and F. Sagues, Phys. Rev. Lett. 76, 3045 (1996).

[13] J. Armero, J. Casademunt, L. Ramírez-Piscina, and J.M. Sancho, Phys. Rev. E 58, 5494 (1998).

[14] C.W. Gardiner, Handbook of Stochastic Methods, 2nd ed. (Springer, New York, 1985).

[15] In [13], Eq. (7) was arrived at by a solvability type analysis assuming a separation of time scales. We will improve this procedure elsewhere, but the more careful analysis does indeed yield the same result (7).

[16] M. Kardar, G. Parisi, and Y.C. Zhang, Phys. Rev. Lett. 56, 889 (1986).

[17] How to obtain this cutoff self-consistently within the KPZ formulation is not completely clear; it obviously is associated with the fact that the terms $\partial^{2} h / \partial \xi^{2}$ and $(\partial h / \partial \xi)^{2}$ are both of the order $1 / t$ at distances $\xi$ of order $\sqrt{t}$.

[18] M. Prähofer and H. Spohn, e-print cond-mat/9910273. 